GUEST EDITORIAL

\title{
Nephrolithiasis (part 2): Diagnosis, management and prevention of recurrent nephrolithiasis
}

\author{
A M Meyers, ${ }^{1}$ FCP (SA), Cert Nephrology (SA), FRCP (Lond) MD; S Naicker, ${ }^{2}$ MB ChB, MRCP (UK), FRCP (Lond), FCP (SA), PhD \\ ${ }^{1}$ Division of Nephrology, Department of Internal Medicine, Faculty of Health Sciences, University of the Witswatersrand, Johannesburg, South Africa \\ ${ }^{2}$ National Kidney Foundation of South Africa, Johannesburg, South Africa
}

Corresponding author: A M Meyers (nkfsa@mweb.co.za)

Work-up of patients with calcium oxalate stones should be restricted to those who have multiple episodes (50\% of stone formers). There are exceptions, such as pilots, fire-workers, people in the armed forces, those with a single kidney and children $(<19$ years old). The most important aspect of therapy is to correct dietary aberrations, i.e. the 'clinic effect'. The four golden rules to correct dietary influences are to maintain an adequate 24-hour urine volume, to restrict salt intake, to avoid red meat and to avoid a diet high in oxalate. In particular, Ceylon tea is contraindicated, as it contains excess oxalate. Drink coffee instead. In patients with hypocitraturia, the drug of choice is potassium citrate, which is the only acceptable urinary alkaliser. Other preparations, such as Urolyte-U, Citrosoda, etc., all have a high sodium content, which will influence urinary calcium excretion. In patients with ongoing hypercalciuria, thiazides are contraindicated in the management of recurrent calcium oxalate stones, and indapamide is the drug of choice. The treatment and follow-up are lifelong. In addition, poor adherence to treatment, coupled with repeated urological procedures, may result in renal functional deterioration and even occasionally renal failure.

S Afr Med J 2021;111(11):1042-1045. https://doi.org/10.7196/SAMJ.2021.v111i11.15992

A detailed diagnostic work-up for renal calculi is beyond the scope of this presentation. Therefore, this presentation is related mainly to calcium oxalate $(\mathrm{CaOx})$ stone formation.

\section{Diagnosis of nephrolithiasis}

Very briefly, conditions such as genetically acquired diseases (e.g. cystinuria, Dent's disease, renal tubular acidosis (RTA), primary hyperparathyroidism, granulomatous hypercalcaemia or, especially, hypercalciuria, autosomal dominant polycystic kidney disease (ADPKD) associated with kidney stones should be referred to the relevant discipline (e.g. urologist, endocrinologist, etc.).

The first principle is to know when a patient with $\mathrm{CaOx}$ stone disease needs a detailed work-up. Therefore, exclusion and inclusion criteria have been formulated.

\section{Exclusion criteria}

A single (first) attack of $\mathrm{CaOx}$ stone disease. Patients with uric acid calculi are also excluded, as are those with calculi associated with infection and/or obstruction.

\section{Inclusion criteria}

The first clinically symptomatic attack of kidney stone disease is included in the metabolic work-up if the radiological investigation depicts multiple stone disease. Patients $<19$ years of age, pilots, fireworkers or armed forces personnel who present with a first stone all require a full metabolic work-up. The same applies to persons with a single kidney, and a first stone in obese patients with diabetes and metabolic syndrome.

The management of renal stones in pregnancy requires specific knowledge of how to handle the situation, and is well dealt with in the review by Meher et al. ${ }^{[1]}$ on renal stones in pregnancy. As the urine volume and mineral content vary vastly during pregnancy, a pathological urinary work-up is contraindicated. In fact, very little work has been done on this subject, and our personal advice is to recommend that the work-up be delayed until 6 months post partum. Of note, $70 \%$ of calcium stones formed during pregnancy consist of calcium phosphate $(\mathrm{CaP})$. These are non-infective in origin, and mandate a full stone work-up post partum. Idiopathic stones consisting of calcium and phosphorus are much more likely to affect kidney function and occasionally result in chronic kidney disease ${ }^{[2]}$ General management and kidney stones in pregnancy is shown in Table 1 .

Point 8 in the table recommends that pregnant patients take an appropriate dose of K-Cit. Although citrate inhibits the crystallisation of $\mathrm{CaP}$, it also alkalinises the urine, which could do more harm than good by resulting in $\mathrm{CaP}$ stone formation, and is therefore not recommended. Thus the main therapy in pregnancy is a very large fluid intake, which will aid spontaneous stone passage. About $70 \%$ of all calcium stones are passed spontaneously during pregnancy owing to the presence of marked urinary tract dilatation. ${ }^{[1]}$

\section{Work-up in secondary causes of $\mathrm{CaOx}$ nephrolithiasis} Although not in the scope of this article, secondary causes are briefly mentioned for the sake of clarity. They include the exclusion of primary hyperparathyroidism, the granulomatous hypercalcaemias, drug-induced RTA, recurrent $\mathrm{CaOx}$ stone formation in patients with $\mathrm{ADPKD}^{[5]}$ and stones associated with inflammatory bowel disorders.

\section{Stone work-up protocols}

- Routine blood tests (Table 2)

- Routine diagnostic radiology (ultrasound or computed tomography scan)

- Dietary work-up - see supplementary material: block 1 (http:// samj.org.za/public/sup/15992.pdf) on dietary assessment.

- Patient information - what to discuss with the patient - see 


\section{Table 1. Nephrolithiasis during pregnancy: Eight essential guideline points}

1. As far as is possible, pregnancy should be planned in patients with $\mathrm{RCaOx}$ stone formation.

2. These patients should, if required by their attending urologist, be stone-free because of the seriousness of possible stone passage during pregnancy.

3. If patients are on either maintenance therapy on thiazides or indapamide, these should be avoided until the postpartum period.

4. If on allopurinol, this agent should be discontinued as some animal studies have indicated fetal toxicity. ${ }^{[3]}$

5. Urinary tract infection is common in pregnancy in patients with recurrent nephrolithiasis and must be carefully monitored and managed.

6. The care team should include, in addition to an informed obstetrician, both the attending urologist and kidney stone nephrologist, if possible.

7. Antenatal visits are guided by the clinical situation but must be more frequent in patients with stones, especially if they have the metabolic syndrome, or are diabetic or have secondary causes of recurrent $\mathrm{CaOx}$ stone formation.

8. Of paramount importance is the necessity to always remain well hydrated, aiming at $2.5 \mathrm{~L}$ (at least) of water per day. Although not studied, it would seem desirable that pregnant women should either commence with or remain on a three times per day appropriate dose of K-Cit with careful monitoring of the urinary $\mathrm{pH}$

$\mathrm{RCaOx}=$ recurring calcium oxalate; $\mathrm{CaOx}=$ calcium oxalate Reproduced with permission from Meyers. ${ }^{[4]}$

\section{Table 2. Routine blood tests ${ }^{\star}$}

\section{Full blood count}

Platelets

Erythrocyte sedimentation rate

C-reactive protein

Albumin

Urea

Serum creatinine

Electrolytes

Estimated glomerular filtration rate

Calcium

Phosphorus

Parathyroid hormone

Aspartate aminotransferase

Gamma glutamyl transferase

Fasting lipid profile

Fasting blood sugar (glucose)

Thyroid-stimulating hormone

Vitamin D (25(OH)D (25-hydroxy vitamin D))

Uric acid

Serum magnesium (not commonly done in South Africa)

Arterial blood gas (only if required)

Other tests (to be specified on request)

${ }^{*}$ Tests included are routine blood tests for urolithiasis management (both diagnostic and

Reproduced with permission from Meyers. ${ }^{[4}$

supplementary material: block 2 (http://samj.org.za/public/ sup/15992.pdf)

- The work-up in a patient with any form of RTA should be done by a nephrologist

- Routine 24-hour urine tests - see supplementary material: block 3 on patient instructions (http://samj.org.za/public/sup/15992.pdf) as well as vital instructions for all chemical pathology laboratories.

There is one more pivotal factor to stress. Neither the doctor nor the dietician should give the patient any stone management advice until the total work-up is complete and the patient has returned for his or her follow-up. Although the reasons are obvious, it is worthwhile to stress them further. If, at the first visit, advice is given to increase fluid intake or to start dietary manipulation (especially on salt intake), the result of the tests will be falsely skewed, making accurate assessment impossible. Important information to be discussed with the patient is included in the supplementary material.

\section{Management and prevention of stones}

Extracorporeal shock wave lithotripsy (ESWL), lithotripsy and surgical interventions are beyond the scope of this article. Other rarer conditions such as management of cystinuria or primary hyperoxaluria (i.e. genetic) and other rare non-genetic disorders of stone formation will be briefly mentioned.

\section{Lifestyle factors contributing to stone formation}

- Fluid intake: this must be aimed at 2.2 - $2.5 \mathrm{~L}$ per day. Apart from water, lemon juice, coffee and herbal or rooibos tea are fluids of choice. No Ceylon tea is allowed. The most valuable study ever published on stone prevention addressed this simple remedy as recently as $1996^{[6]}$ The frequency of non-adherence and reinforcement of adequate hydration must continue indefinitely.

- Dietary assessment and advice: this is presented in more detail in the appendix. Apart from the assessment of a skilled dietician, the doctor should also become acquainted with the patient's food and eating habits.

- Of importance is to train the 'stone dieticians' and attending medical staff never to give advice to the patient until all the tests and work-up are complete. This also dictates that the doctor will have seen the results of the tests, which will then be discussed with the patient. Failure to carry out these instructions will be the cause of many errors. An important example of this is the assessment of fluid intake. If the patient is instructed by the doctor or dietician to increase fluid intake or to avoid certain foods, the assessment will be false. The initial two to three 24-hour urine work-up assessments yield vital information of the true fluid balance between intake and output.

- In obese patients, weight loss and exercise must be repeatedly advised by the dietician, making follow-up assessments essential.

\section{Medications}

- The use of K-Cit: in practice, it is common to come across stone patients who are advised by their physicians to go onto citrate therapy without justification. Although hypocitraturia is common, about $50 \%$ of patients will not have this condition, and therefore urinary alkalisation with all its attending hazards could well occur. ${ }^{[1,2]}$ Another common problem seen in practice is that other urinary alkalisers have potential problems. The commercial products Urolite U, CitroSoda and sodium bicarbonate all contain significant quantities of sodium. ${ }^{[7]}$ In stone formers, this increased quantity of sodium will result in increased sodium excretion, increasing the theoretical chance of treatment failure with new stone formation. Simple K-Cit is the treatment of choice, ${ }^{[8,9]}$ and is inexpensive. The downside of $\mathrm{K}$-Cit ingestion is the bad taste, which can be eliminated by diluting 
Table 3. Urinary oxalate excretion ( $\mu \mathrm{mol} / 24$ hour) after 'standard' diets

\begin{tabular}{|c|c|c|c|c|c|}
\hline \multirow[b]{2}{*}{ Variable } & \multicolumn{3}{|c|}{ Black } & \multicolumn{2}{|c|}{ White } \\
\hline & Female & Male (study 1$)^{*}$ & Male (study 2$)^{\star}$ & Female & Male \\
\hline$n$ & 19 & 12 & 22 & 17 & 12 \\
\hline Mean (SD) & $244(127)$ & 239 (135) & $271(121)$ & $194(78)$ & $181(164)$ \\
\hline
\end{tabular}

Table 4. Clinical points to remember

Clinical aspect
RCaOx stone presentation
Use of allopurinol
CT scan diagnosis and endo-uroscopic stone removal procedures
Remember the host of secondary and the large list of drug causes
of nephrolithiasis
High salt intake

The most important dietary advice to all stone formers (and everyone else)

General scientific advice behind the overall dietary pattern

\section{Points to remember/interpretation}

Citrate is not the panacea for all $\mathrm{CaOx}$ or $\mathrm{CaP}$ nephrolithiasis treatment.

Subclinical magnesium deficiency states are dangerous and found more in children. Also found in patients on chronic loop diuretics.

This is safe and even important in $\mathrm{CaOx}$ stone formation in normouricosuria patients.

Remember that Randall's plaques are not stones. Patient safety is of paramount importance.

Varied pathogenesis and treatment. Recognition of and treatment of cause and/or discontinuation of offending drug.

Of paramount importance in causing $\mathrm{RCaOxSF}$ and can result in serious osteoporosis. Can only be diagnosed with 24-hour urine examination. Eat a little of everything and a lot of nothing.

Note: When on a weight-reducing diet, this does not apply and only kicks in as maintenance when the target weight has been reached.

Diet imposes patterns of metabolism upon the organism. ${ }^{[17]}$

$\mathrm{RCaOx}=$ recurring calcium oxalate; $\mathrm{CaOx}=$ calcium oxalate; $\mathrm{CaP}=$ calcium phosphate; $\mathrm{Mg}=$ magnesium; $\mathrm{CT}=$ computed tomography; $\mathrm{RCaOxSF}=$ recurring calcium oxalate stone formation Reproduced with permission from Meyers. ${ }^{[4]}$

$10-15 \mathrm{~mL}$ of the solution in a glass of water, taken three times a day (not less), which will also help ensure adequate hydration.

- Hypomagnesaemia/hypomagnesuria: although magnesium is an important inhibitor of stone formation, there is no clinical or experimental basis to recommend it in the absence of low magnesium levels. Most cases of low magnesium are found in children, or the very occasional adult. The most commone magnesium tablet used in South Africa (Slow-Mag) is contraindicated in stone therapy because it consists of magnesium chloride. Magnesium oxide or magnesium hydroxide are the medications of choice for both efficacy and cost-effectiveness. ${ }^{[10]}$

- Hypernatrituria: at the time of writing, the 'true' role of excess dietary salt has not yet been adequately assessed. If patients were compliant enough to decrease their dietary salt intake so as to ensure $80-100 \mathrm{mg}$ of daily sodium loss, most patients with so-called 'idiopathic hypercalciuria' might well have completely normal urinary biochemistry and would cease the formation of new $\mathrm{CaOx}$ calculi. However, this degree of long-term dietary salt restriction is probably nothing but a pipe dream, because lifestyle changes with salt restriction are so difficult to attain. The treatment of other causes of the hypercalciurias is dealt with below.

- Hypercalciurias: there are three subtypes of hypercalciuria. (i) True (primary) renal hypercalciuria - a very rare disease. Some of these patients may be associated with urinary phosphate excretion changes, resulting in a raised level of 1.25 dihydroxy vitamin D3 and stone formation. (ii) Salt-induced hypercalciuria. (iii) Secondary hypercalciuria associated with hyperparathyroidism, sarcoidosis, other granulomas, RTA and vitamin D excess. Secondary hypercalciuria patients are managed according to the underlying cause. Patients falling into primary and salt-induced hypercalciuria are usually treated with thiazide diuretics. In fact, thiazide diuretics are completely contraindicated, which has been clearly demonstrated in previous publications. ${ }^{[1,11]}$ The doses of thiazide required for the treatment of hypercalciuria (25 - $50 \mathrm{mg}$ per day) are in the toxic range, and their hypocalciuric effect disappears after 6 months to 1 year of therapy. The treatment of choice in these patients is indapamide, and the urinary calciumlowering effects continue indefinitely. ${ }^{[1,12]}$

- Urinary oxalate excretion: The present laboratory normal 24-hour urinary oxalate excretion range is totally inaccurate. This criticism applies especially to the so-called mild metabolic hyperoxalurias (MMHO). Standard diets are required before MMHO can be verified. In addition, laboratories should report the results, as the excretion of urine oxalate in $\mu \mathrm{mol}$ per 24 hours and not $\mu \mathrm{mol}$ per litre. ${ }^{[13,14]}$ Urinary oxalate excretion has been measured in previous studies. ${ }^{[15,16]}$ In summary, the normal ranges that were found in these studies are shown in Table 3.

There are two more points to be made. Firstly, there was no difference in the excretion of oxalate between males and females, or between black and white patients. Secondly, because oxalate is so ubiquitous and is found in most foodstuffs (particularly red meat), the practical normal excretion on a low oxalate diet has been regarded as $350 \mu \mathrm{mol}$ per 24 hours. If the dietary standardisation is accurate and the oxalates are above this level, the two methods used in treatment are to increase the dietary calcium ingestion (which binds to oxalate and prevents absorption), or to add pyridoxine, which is only effective in a small minority. ${ }^{[16]}$

However, causes of secondary (i.e. acquired) hyperoxaluria, such as is seen in inflammatory bowel diseases or after barosurgery, 
are significantly higher than the normal range $(600-1500 \mu \mathrm{mol}$ per 24 hours). In patients with hereditary oxalosis, the levels are considerably higher than the above, and reach as high as $3000 \mu \mathrm{mol}$ per day.

Patients with kidney stone disease are usually worked-up using a 'best practice available' basis. They have one 24-hour urine work-up, urine cultures where necessary, relevant blood tests and radiology and, if the stone does not pass spontaneously after fluid load, extracorporeal shockwave lithotripsy or laser therapy are considered. For long-term maintenance, patients are normally referred to a general practitioner. In spite of the skills and good intentions of the attending physicians, the above mode of therapy is not in the best interest of the patient, nor is it cost-effective. However, any longterm and detailed stone management programme is very difficult to conceive without the services of a multidisciplinary team.

In summary, the main aims of this article have been to educate all interested personnel on what could and should be done for patients with recurrent renal calculi, i.e. the 'office' approach. The most important clinical points to remember are shown in Table 4, and the most important management aspects are shown in the included appendices.

Declaration. None.

Acknowledgements. None.

Author contributions. Equal contributions.

Funding. None.

Conflicts of interest. None.

1. Meher S, Gibbons N, DasGupta R. Renal stones in pregnancy. Obstet Med 2014;7(3):103-110. https://doi org/10.1177/1753495X14538422

2. Coe F. Calcium phosphate stones: Causes and prevention. University of Chicago, 2021. https./ kidneystones.uchicago.edu/idiopathic-calcium-phosphate-stone-formers/ (accessed 30 June 2021).
3. Division of Clinical Pharmacology, Faculty of Health Sciences, University of Cape Town, Rossiter D, ed. South African Medicines Formulary. 10th ed. Cape Town: Health and Medical Publishing Group, 2012

4. Meyers AM. The pivotal role of a kidney stone clinic in the management and prevention of recurrent calcium oxalate nephrolithiasis. Wits Institutional Repository on DSpace, 2020. http://wiredspace. wits.ac.za/browse?authority=77f47495-e38c-4841-9362-428505767a29\&type=author $\quad$ (accessed 7 July 2021)

5. Torres JA, Rezaei M, Broderick C, et al. Crystal deposition triggers tubule dilation that accelerates cystogenesis in polycystic kidney disease. J Clin Investig 2019;129(10):4506-4522. https://doi, org/10.1172/JCI128503

6. Borghi L, Meschi T, Amato F, Briganti A, Novarini A, Giannini A. Urinary volume, water and recurrences in idiopathic calcium nephrolithiasis: A 5-year randomised prospective study. J Urol 1996;155(3):839-843. https://www.ncbi.nlm.nih.gov/pubmed/8583588 (accessed 15 October 2021).

7. Krieger NS, Asplin JR, Frick KK, et al. Effect of potassium citrate on calcium phosphate stones in a model of hypercalciuria. J Am Soc Nephrol 2015;26(12):3001-3008. https://doi.org/10.1681/ in a model of hyperc.

8. Whalley NA, Meyers AM, Martins M, Margolius LP. Long-term effects of potassium citrate therapy on the formation of new stones in groups of recurrent stone formers with hypocitraturia. $\mathrm{Br} \mathrm{J}$ Urol 1996;78(1):10-14. https://doi.org/10.1046/j.1464-410x.1996.09852.x

9. Pak CY, Fuller C, Sakhaee K, Preminger GM, Britton F. Long-term treatment of calcium nephrolithiasis with potassium citrate. J Urol 1985;134(1):11-19. https://doi.org/10.1016/s0022$5347(17) 46962-\mathrm{x}$

10. Johansson G, Backman U, Danielson BG, Fellström B, Ljunghall S, Wikström B. Effects of magnesium hydroxide in renal stone disease. J Am Coll Nutr 1982;1(2):179-185. https://doi.org/10 $1080 / 07315724.1982 .10718985$

11. Martins MC, Meyers AM, Whalley NA, Margolius LP, Buys ME. Indapamide (Natrilix): the agent of choice in the treatment of recurrent renal calculi associated with idiopathic hypercalciuria. Br J Urol 1996;78(2):176-180. https://doi.org/10.1046/j.1464-410x.1996.00633.x

12. Meyers AM, Martins M, Kung C, Bellingan A, Margolius LP. The efficacy and safety of indapamide compared with hydrochlorothiazide in the treatment of calculi associated with renal hypercalciuria. Proceedings of the Fifth European Urolithiasis Symposium, Manchester UK, 21 - 23 April 1994. In: Rao PN, Kavanagh IP. Tiselius H-G, editors. Urolithiasis Consensus and Controversies. Manchester: Lithotriptor Unit, South Manchester University Hospitals, 1995:281-283.

13. Holmes RP, Kennedy M. Estimation of the oxalate content of foods and daily oxalate intake. Kidney Int 2000;57(4):1662-1667. https://doi.org/10.1046/j.1523-1755.2000.00010.x

14. Harvard TH Chan School of Public Health Nutrition Department. Directory listing of /health/ Oxalate/files. https://regepi.bwh.harvard.edu/health/Oxalate/files (accessed12 December 2018).

15. Whalley NA, Moraes MF, Shar TG, Pretorius SS, Meyers AM. Lithogenic risk factors in the urine of black and white subjects. Br J Urol 1998;82(6):785-790. https://doi.org/10.1046/j.1464410x.1998.00877.x

16. Whalley NA, Martins MC, Van Dyk RC, Meyers AM. Lithogenic risk factors in normal black volunteers, and black and white recurrent stone formers. BJU Int1999;84(3):243-248. https://doi. org/10.1046/j.1464-410x.1999.00170.x

17. Gillman J, Gillman T. Perspectives in Human Malnutrition: A Contribution to the Biology of Disease from a Clinical and Pathological Study of Chronic Malnutrition and Pellagra in the African. New York: Grune \& Stratton, 1951

Accepted 29 July 2021 